http://dx.doi.org/10.15407/ukrbotj72.01.050

Yu.V. SHCHERBAKOVA, V.V. DZHAGAN

Educational and Scientific Centre «Institute of Biology», Taras Shevchenko National University of Kyiv

Volodymyrska St., 64, Kyiv, 01601, Ukraine

dzhagan@yahoo.com

\title{
SCUTELLINIA TORRENTIS (PYRONEMATACEAE, PEZIZALES), A NEW FOR UKRAINE SPECIES FROM CARPATHIAN BIOSPHERE RESERVE
}

\begin{abstract}
Shcherbakova Yu.V., Dzhagan V.V. Scutellinia torrentis (Pyronemataceae, Pezizales), a new for Ukraine species from Carpathian Biosphere Reserve.- Ukr. Bot. J. - 2015. - 72(1): 50-54.

A new for Ukraine species, Scutellinia torrentis (Rehm) T. Schumach. (Pyronemataceae, Pezizales), is reported. The species was collected in the Svydovetskyi Mountain Range of Carpathian Biosphere Reserve. A brief description, localities, data on general distribution, taxonomic notes, photomicrographs and original illustrations are provided.
\end{abstract}

Ke y words: Ascomycota, operculate discomycetes, new records, Ukraine.

\section{Introduction}

Despite over hundred years of history of mycological research in the Ukrainian Carpathians, diversity of some taxa, including the genus Scutellinia (Cooke) Lambotte, has not been studied well. According to available literature data (Смицкая, 1980; Namyslowski, 1909; Andrianova et al., 2006; Дудка, Джаган, 2011), so far only three species of this genus have been recorded in the Ukrainian Carpathians. The first Scutellinia species in the region was recorded in the early $20^{\text {th }}$ century by Namyslowski (1909). He registered S. scutellata (L.) Lambotte in Prykarpattya (Ivano-Frankivsk Region). In 1969, L. Smyk collected S. umbrorum (Fr.) Lambotte from the Uzhok Pass (Velykobereznyanskyi District, Zakarpattia Region) (Andrianova et al., 2006). During our examination of mycobiota in Carpathian Biosphere Reserve in 2006, S. trechispora (Berk. \& Broome) Lambotte was found (Дудка, Джаган, 2011). Since 2009 until recently we studied fungal diversity in the Svydovetskyi Mountain Range of the Carpathian Biosphere Reserve. Consequently, such species as $S$. cejpii (Velen.) Svrcek, S. crinita (Bull.) Lambotte, S. crucipila (Cooke \& W. Phillips) J. Moravec, S. pseudotrechispora (J. Schröter) Le Gal, S. olivascens (Cooke) Kuntze, S. scutellata (L.) Lambotte, S. subhirtella Svrcek, and $S$. trechispora (Berk. \& Broome) Lambotte were registered. Some of these records were published earlier (Щербакова, Джаган, 2013; Dzhagan, Scherbakova, 2013). The present article reports another species new for Ukraine, S. torrentis (Rehm) T. Schumach. The specimens were collected in 2011-2012 during our my-

(C) YU.V. SHCHERBAKOVA, V.V. DZHAGAN, 2015 cological survey in the Svydovetskyi Mountain Range (Rakhiv District, Zakarpattya Region).

\section{Materials and methods}

Macroscopic descriptions of the collected specimens are based on fresh ascomata. Microscopic features are described from dried material mounted in $\mathrm{H}_{2} \mathrm{O}, 3 \%$ $\mathrm{KOH}$ solution, Congo Red solution, and Cotton Blue in lactic acid, using an Ulab XY-B2T light microscope and Canon PC 1089 PowerShot G6 camera. Analysis of general distribution is based on the data from published sources (Schumacher, 1990; Gonzáles et al., 1997; Eriksson, 2011; Mihal et al., 2011; Olariaga, Hansen, 2011; Jeannerot, 2013; Greaves, 2014) and databases available through the Internet, including The Catalogue of Life (Roskov et al., 2014) and Belgian Species List (http://www.species.be/en/43787). Collected specimens are preserved in Herbarium of the Taras Shevchenko National University of Kyiv (KWU). Species of fungi are arranged following Index Fungorum (Kirk, 2003).

\section{Results and discussion}

A brief description of the species, associated substrates, localities, information on general distribution, taxonomic notes and original illustrations are provided below.

Scutellinia torrentis (Rehm) T. Schumach., Opera Botanica 101: 97. 1990. - Lachnea torrentis Rehm, Annales Mycologici 5(3): 212. 1907. - Scutellinia convexa (Velen.) Svrček, Česká Mykologie 25(2): 83. 1971. Scutellinia marginata Gamundí, Flora Criptogámica de Tierra del Fuego 10(3): 94.1975 ( Fig. 1, 2.) 

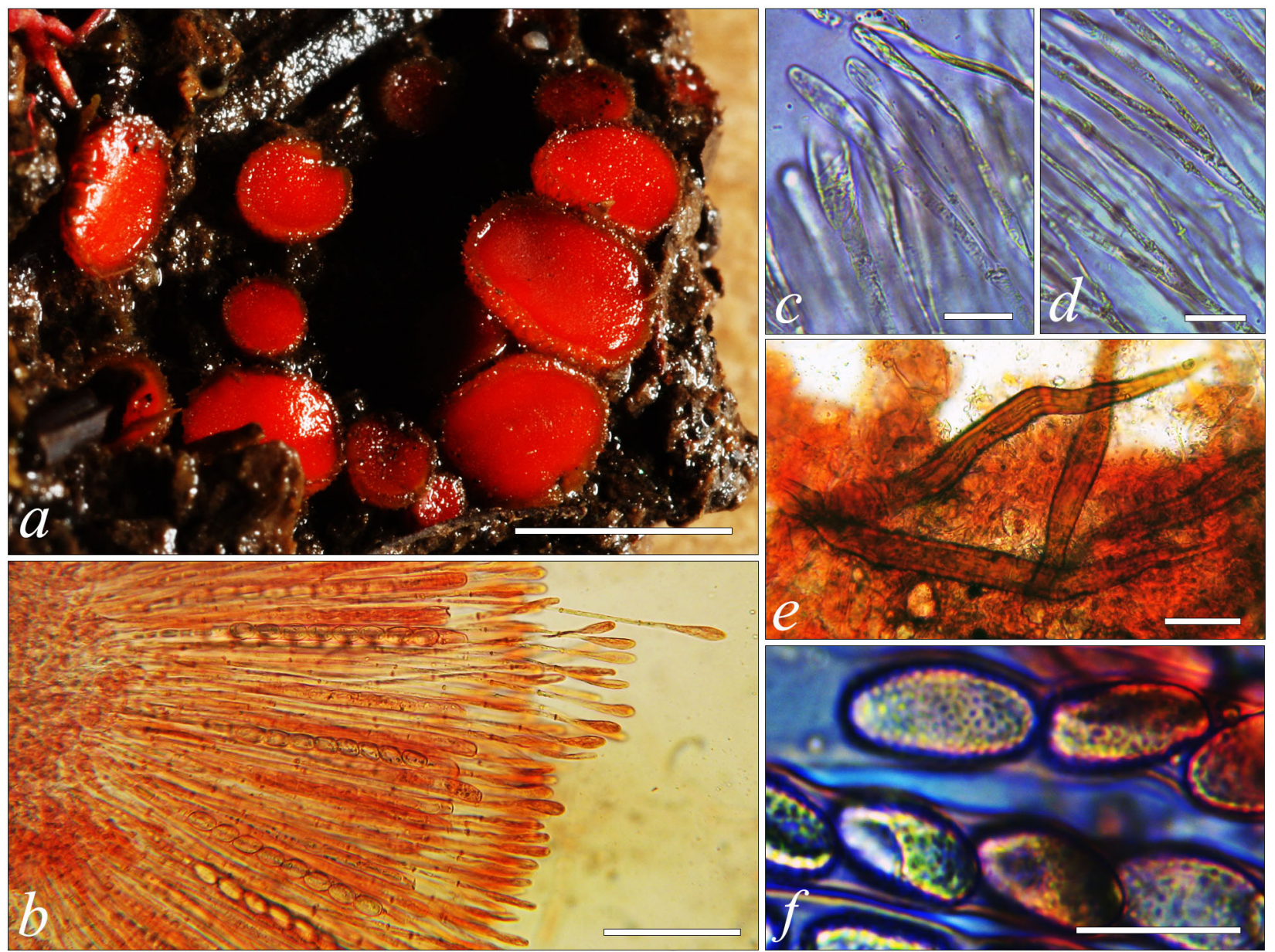

Fig. 1. Scutellinia torrentis: $a$ - apothecia; $b$ - fragment of hymenial layer; $c, d$ - paraphyses; $e$ - hairs; $f$ - fragment of ascus with spores. Bars: $a-3 \mathrm{~mm} ; b-50 \mu \mathrm{m} ; c, d, f-20 \mu \mathrm{m} ; e-30 \mu \mathrm{m}$

Fig. 2. Scutellinia torrentis: $a$ - ascus with spores; $b-$ paraphyses; $c$ - hairs; $d$ - spores. Bars: $a, b, c-20$ $\mu \mathrm{m} ; d-10 \mu \mathrm{m}$

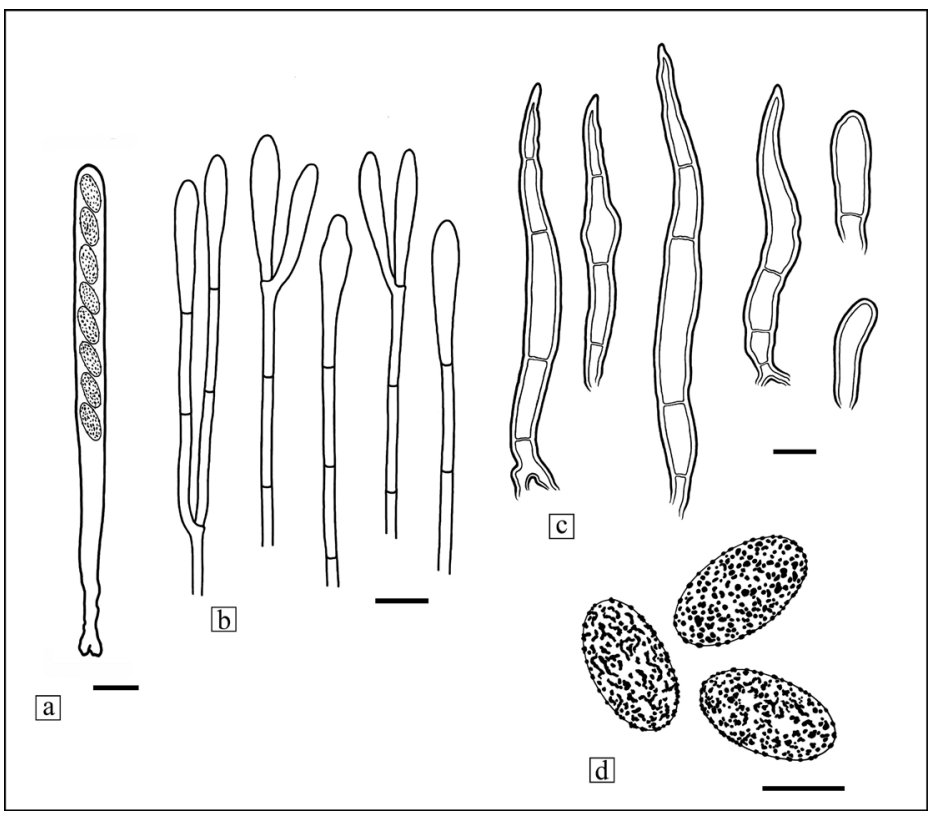




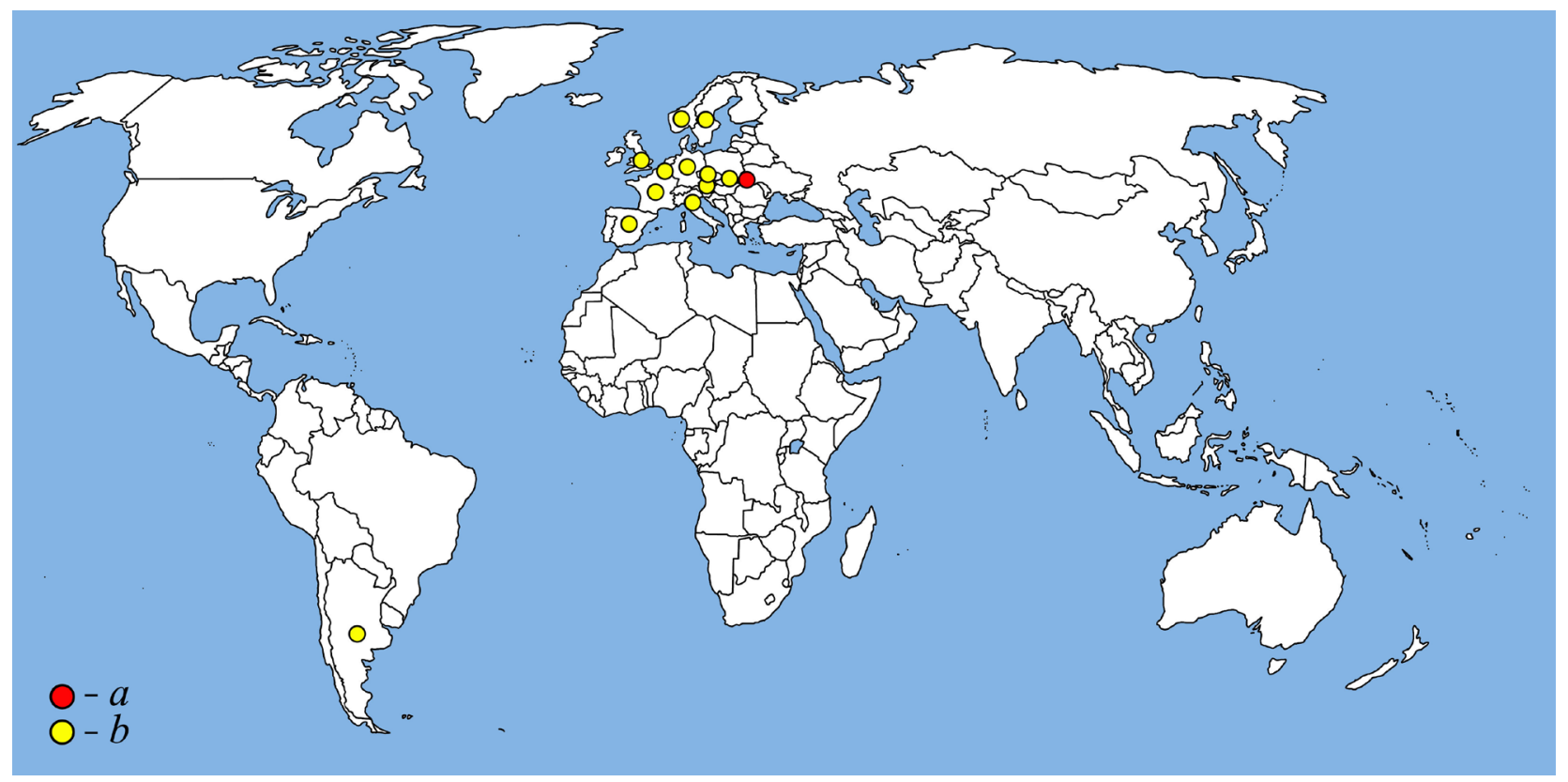

Fig. 3. Worldwide distribution map of Scutellinia torrentis: $a$ - Ukrainian record; $b$ - sites reported by other authors

Apothecia gregarious, disc-shaped, 2-8 $\mathrm{mm}$ in diam., with convex hymenium and slightly raised margin. Hymenium red to brownish red. Outer surface and margin densely covered by short, brown hairs. Ectal excipulum of angular to globose cells, $15-60 \times 20-50$ $\mu \mathrm{m}$, outermost layers with elongated cells turning out perpendicularly to the apothecial surface, whole zone 175-350 $\mu \mathrm{m}$ thick. Hairs not differentiated into marginal and receptacular, 100-320 $(400) \times 15-35 \mu \mathrm{m}$, of more or less even length, usually $200-250 \times 20 \mu \mathrm{m}$, 1-4-septate, pointed, flexuous and uneven, often constricted at the septae, thick-walled, brownish, with a long and attenuated unbranched or bi-tri-furcate narrow base, wall 3-6 $\mu \mathrm{m}$ thick. Between the stiff, brownish hairs are dense clusters of blunt, short, broad, subhyaline «hyphoid hairs» forming raised margin. Asci cylindric, 190-250 × 13-19 $\mu \mathrm{m}$, gradually narrowing toward short, pleurorhynchous base. Ascospores oblong ellipsoidal, $15.5-19.2 \times 7.8-11.5 \mu \mathrm{m}$, multiguttulate. Spore sculpturing consisting of rounded to elongated warts, $0.3-1.4 \mu \mathrm{m}$ wide, up to $0.6 \mu \mathrm{m}$ high. Warts usually confluent and interconnecting to short ridges forming incomplete pseudo-reticulum, warts unevenly distributed on spore wall. Outermost spore layer loosening like envelope in heated lactic acid, floating around spore. Paraphyses $2.5-3.5 \mu \mathrm{m}$ wide, straight, septate, enlarged above to $8-12 \mu \mathrm{m}$, partly branched from api- cal, middle and distal parts (Schumacher, 1990; Hansen, Knudsen, 2000).

Specimen examined: Zakarpattia (Transcarpathian) Region, Rakhiv District; ski complex Dragobrat (18 km from Yasynia settlement), on sandy soil, associated with S. crucipila (Cooke \& W. Phillips) J. Moravec, 5 July 2011, Yu. Shcherbakova; Svydovets Mountain Range of Carpathian Biosphere Reserve, in the vicinity of Dragobrat, mixed forest with domination of Picea $a b$ ies, near the creek, on wet soil, 23 September 2012, Yu. Shcherbakova.

General distribution (Fig. 3.).

Europe: Austria (Damon, 2005), Belgium (http:// www.species.be/en/43787), Czech Republic (Schumacher, 1990), France (Jeannerot, 2013), Germany (Schumacher, 1990; Roskov et al., 2014), Italy (Roskov et al., 2014), Norway (Schumacher, 1990), Slovakia (Mihal et al., 2011), Spain (Gonzáles et al., 1997), Sweden (Eriksson, 2011; Olariaga, Hansen, 2011), United Kingdom (Greaves, 2014); South America: Argentina (Schumacher, 1990).

Notes: Humus saprotroph (Schumacher, 1990). This species grows in spruce and mixed beech and spruce forests on alkaline and acidic soils (Mihal et al., 2011); it is typical for modified areas, soggy, then dried, such as forest ruts (Jeannerot, 2013). In summer-autumn (Hansen, Knudsen, 2000). 
The gregarious growth, convex red hymenium, short flexuous hairs, and ascospores with amoeboid warts and crests on the outermost wall layer loosening in lactic acid, characterize this species (Schumacher, 1990).

\section{Conclusion}

The studied species is included in sect. Minutae Svr. series Minutae by Schumacher (1990), based on such characteristics as ascospores with verrucose to pustulocristate ornamentation and the outermost wall layer readily separating in lactic acid.

It should be noted that $S$. torrentis is considered a rare species. It is listed in the Red List Candidates of the European Council for the Conservation of Fungi (http://www.wsl.ch/eccf/candlist-subtotals.xls). The reported locality represents the easternmost point of the known up to now distribution range of this species. For conclusions on the occurrence of this rare species in Ukraine, further observations in the Carpathians are required.

\section{Acknowledgements}

The authors are grateful to Beñat Jeannerot (Lycée Agricole Technologique Privé, France) for helpful advices on the identification of the species.

\section{REFERENCES}

Дудка I.O., Джсаган В.В. Заповедные леса Украинских Карпат как рефугиум для сохранения редких видов миксомицетов и грибов // Роль ботанических садов и охраняемых природных территорий в изучении и сохранении разнообразия растений и грибов. Сб. материалов Всеросс. научн. конф. с междунар. участием (13-16 окт. 2011 г., Ярославль). - Ярославль: Изд-во ЯГПУ, - С. 173-175.

Смицкая М.Ф. Флора грибов Украины. Оперкулятные дискомицеты. - Киев: Наук. думка, - 224 с.

Щербакова Ю., Джаган В. Карботрофні дискоміцети Українських Карпат // Вісн. Львів. ун-ту. Сер. біол. - 2013. - 63. - С. 118-126.

Andrianova T.V., Dudka, I.O., Hayova V.P., Heluta V.P., Isikov V.P., Kondratiuk S.Ya., Krivomaz T.I., Kuzub V.V., Minter D.W., Minter T.J., Prydiuk M.P., Tykhonenko Yu.Ya. (2006 onwards) / Ed. D.W. Minter \& I.O. Dud- ka. Fungi of Ukraine. Website. Version 1.00. www.cybertruffle.org.uk/ukrafung/eng (21.02.2015)

Damon $W$. Die Pilzflora des Schwingrasen-Moorwaldes am Krottensee (Gmunden, Oberrosterreich) // Osterr. Z. Pilzk. - 2005. - 14. - P. 123-190.

Dzhagan V.V., Scherbakova Yu.V. New for Ukraine species of Scutellinia (Pyronemataceae, Pezizales) from the Svydovets Mountain Range (Carpathian Biosphere Reserve) // Укр. ботан. журн. - 2013. - 70(3). - P. 405-410.

Eriksson O.E. Senaste nytt om listan över Svenska Ascomyceter // Svensk Mykologisk Tidskrift. - 2011. - 32(2). - P. 23-26.

Gonzáles $V$., Esteve-Raventós $F$., Arenal Yague $F$., Villarreal $M$. Contribución al estudio taxonómico de los macromicetos de zonas higroturbosas alpinas y subalpinas del Pirineo central // Lucas Mallada: revista de ciencias. - 1997. - 9. - P. 71-90.

Greaves $M$. An introduction to Scutellinia and a new British species: $S$. torrentis // Field Mycology. - 2014. - 15(1). - P. 9-11.

Hansen L., Knudsen H. Nordic Macromycetes. Vol. 1 (Ascomycetes). - Copenhagen: Nordsvamp., 2000. - 309 p.

Jeannerot $B$. Contribution à l'étude du genre Scutellinia (Pezizales). III: Quelques espèces récoltées au cours de la session Ascomycetes «zone alpine» 2013 de Beaufort // Ascomycete. org. - 2013. - 5(5). - P. 159-170.

Kirk P.M. (2003 onwards) Index Fungorum - The global nomenclatural database for fungi. http://www.indexfungorum.org (21.02.2015)

Mihal I., Glejdura S., Blanar D. Makromycety (Zygomycota, Ascomycota, Basidiomycota) v masive Kohuta (Stolicke vrchy) // Reussia. - 2011. - 6. - P. 1-44.

Namysłowski B. Zapiski grzyboznawcze z Krakowa, Gorlic i Czarney Hory // Sprawozdanie Komisji Fizjograficzney Polskiej Akademii Umiejętności. - 1909. - 43(2). - P. 3-30.

Olariaga I., Hansen K. New and noteworthy records of Pezizomycetes in Sweden and the Nordic countries // Karstenia. - 2011. - 51. - P. 1-16.

Roskov Y., Kunze T., Orrell T., Abucay L., Paglinawan L., Culham A., Bailly N., Kirk P., Bourgoin T., Baillargeon G., Decock W., De Wever A., Didžiulis V. (2014 onwards). Species 2000 \& ITIS Catalogue of Life, 2014 Annual Checklist. Digital resource at www.catalogueoflife.org/ annual-checklist/2014 (21.02.2015)

Schumacher T. The genus Scutellinia (Pyronemataceae) // Opera Botanica. - 1990. - 101. - P. 1-107.

Recommended for publication by V.P. Heluta 
Щербакова Ю.В., Джаган В.В. Scutellinia torrentis (Pyronemataceae, Pezizales) - новий для України вид 3 території Карпатського біосферного заповідника. -Укр. ботан. журн. - 2015. - 72(1): 50-54.

Навчально-науковий центр «Інститут біології», Київський національний університет імені Тараса Шевченка, Україна

Повідомляється про знахідку на території Свидовецького масиву Карпатського біосферного заповідника нового для України виду роду Scutellinia (Pyronemataceae, Pezizales) $-S$. torrentis (Rehm) T. Schumach. Подано його короткий опис, місцезнаходження, інформацію щодо загального поширення, таксономічні примітки та оригінальні ілюстрації.

Ключов і слов а: Ascomycota, оперкулятні дискоміцети, нові знахідки, Україна.
Щербакова Ю.В., Джаган В.В. Scutellinia torrentis (Pyronemataceae, Pezizales) - новый для Украины вид с территории Карпатского биосферного заповедника. - Укр. ботан. журн. - 72(1): 50-54.

Учебно-научный центр «Институт биологии», Киевский национальный университет имени Тараса Шевченко, Украина

Сообщается о находке на территории Свидовецкого массива Карпатского биосферного заповедника нового для Украины вида рода Scutellinia (Pyronemataceae, Pezizales) - S. torrentis (Rehm) T. Schumach. Приведены его краткое описание, местонахождения, информация об общем распространении, таксономические примечания и оригинальные иллюстрации.

Ключе в ы е слов а: Ascomycota, оперкулятные дискомицеты, новые находки, Украина.

\section{НОВІ ВИДАННЯ}

Придюк Н.П. Флора грибов Украины. Больбитиевые и коприновые грибы / Ред. И.О. Дудка. - Киев: ООО НПП Интерсервис, 2015. - 598 с.

Придюк М.П. Флора грибів України. Больбитієві та копринові гриби / Ред. И.О. Дудка. - К.: ТОВ НПП Інтерсервіс, 2015. - 598 c.

У монографії узагальнені оригінальні та літературні дані про больбитієві та копринові гриби України. Наведено відомості щодо їх морфології, анатомії, циклів розвитку, еколого-біологічних особливостей та систематики. Вміщено найновішу інформацію про значення цих грибів у природі та господарстві. Вказано ключі для визначення родин, родів, внутрішньородових таксонів, видів і різновидів. Цікавими $є$ матеріали як про відомі в Україні види, так і про деякі потенційно можливі для ії території. Для кожного таксона подано синоніміку, іконографію, загальний опис макро- та мікроскопічної будови, відомості про місцезростання, поширення в Україні та світі, критичні примітки. Для видів, виявлених в Україні, вміщено оригінальні ілюстрації.

Для мікологів, співробітників природоохоронних об'єктів, викладачів і студентів вишів. 